\title{
Pleural biomarkers in diagnostics of malignant pleural effusion: a narrative review
}

\author{
Man Zhang ${ }^{1}$, Li Yan ${ }^{2}$, Giuseppe Lippi ${ }^{3}$, Zhi-De Hu ${ }^{4}$ \\ ${ }^{1}$ Department of Thoracic Surgery, the Affiliated Hospital of Inner Mongolia Medical University, Hohhot, China; ${ }^{2}$ Department of Respiratory and \\ Critical Care Medicine, the Affiliated Hospital of Inner Mongolia Medical University, Hohhot, China; ${ }^{3}$ Section of Clinical Biochemistry, University \\ of Verona, Verona, Italy; ${ }^{4}$ Department of Laboratory Medicine, the Affiliated Hospital of Inner Mongolia Medical University, Hohhot, China \\ Contributions: (I) Conception and design: ZD Hu; (II) Administrative support: L Yan, M Zhang; (III) Provision of study materials or patients: None; (IV) \\ Collection and assembly of data: M Zhang; (V) Data analysis and interpretation: M Zhang, L Yan; (VI) Manuscript writing: All authors; (VII) Final \\ approval of manuscript: All authors. \\ Correspondence to: Zhi-De Hu. Department of Laboratory Medicine, the Affiliated Hospital of Inner Mongolia Medical University, Hohhot, China. \\ Email: hzdlj81@163.com.
}

\begin{abstract}
Although cytology and pleural biopsy of pleural effusion (PE) are the gold standards for diagnosing malignant pleural effusion (MPE), these tools' diagnostic accuracy is plagued by some limitations such as low sensitivity, considerable inter-observer variation and invasiveness. The assessment of PE biomarkers may hence be seen as an objective and non-invasive diagnostic alternative in MPE diagnostics. In this review, we summarize the characteristics and diagnostic accuracy of available PE biomarkers, including carcinoembryonic antigen (CEA), neuron-specific enolase (NSE), carbohydrate antigens 125 (CA125), carbohydrate antigen 19-9 (CA19-9), carbohydrate antigen 15-3 (CA15-3), a fragment of cytokeratin 19 (CYFRA 21-1), chitinase-like proteins (CLPs), vascular endothelial growth factor (VEGF) and its soluble receptor, endostatin, calprotectin, cancer ratio, homocysteine, apolipoprotein E (Apo-E), B7 family members, matrix metalloproteinase (MMPs) and tissue-specific inhibitors of metalloproteinases (TIMPs), reactive oxygen species modulator 1 (Romo1), tumor-associated macrophages (TAMs) and monocytes, epigenetic markers (e.g., cell-free microRNA and mRNA). We summarized the evidence from systematic review and meta-analysis for traditional tumor markers' diagnostic accuracy. According to the currently available evidence, we conclude that the traditional tumor markers have high specificity (around 0.90) but low sensitivity (around 0.50). The diagnostic accuracy of novel tumor markers needs to be validated by further studies. None of these tumor biomarkers would have sufficient diagnostic accuracy to confirm or exclude MPE when used alone. A multi-biomarker strategy, also encompassing the use of artificial intelligence algorithms, may be a valuable perspective for improving the diagnostic accuracy of MPE.
\end{abstract}

Keywords: Tumor marker; malignant pleural effusion (MPE); review; diagnosis

Submitted Oct 12, 2020. Accepted for publication Jan 29, 2021.

doi: $10.21037 /$ tlcr-20-1111

View this article at: http://dx.doi.org/10.21037/tlcr-20-1111

\section{Introduction}

Pleural effusion (PE) is a relatively common sign in clinical practice. It can be caused by various conditions, including malignant pleural effusion (MPE), tuberculous pleural effusion (TPE), parapneumonic effusion (PPE), heart failure (HF), and others (1). PE cytology and thoracentesis with pleural biopsy are the gold standards for diagnosing MPE (1). However, both of these techniques have limitations. Low sensitivity is perhaps the major drawback of $\mathrm{PE}$ cytology, though its specificity is very close to $100 \%$ (2). Like PE cytology, the pleural biopsy also has very high specificity (i.e., approximating 100\%), but is invasive, may be vulnerable to sampling error, is highly subjective, and 
Table 1 Diagnostic accuracy of traditional tumor markers for malignant pleural effusion: evidence from meta-analysis

\begin{tabular}{|c|c|c|c|c|c|c|c|c|c|}
\hline Author & Year & Tumor marker & MPE/BPE & $\mathrm{N}$ & Model & $A \cup C$ & Sensitivity $(95 \% \mathrm{Cl})$ & Specificity (95\% Cl) & PB \\
\hline Shi (7) & 2008 & CEA & $2,834 / 3,251$ & 45 & REM & 0.77 & $0.54(0.52-0.55)$ & $0.94(0.93-0.95)$ & Yes \\
\hline Gu (8) & 2007 & CEA & $1,404 / 1,543$ & 15 & DLM & 0.77 & $0.46(0.43-0.49)$ & $0.97(0.96-0.98)$ & NR \\
\hline Nguyen (6) & 2015 & NSE & NR & 7 & REM & 0.84 & $0.61(0.26-0.96)$ & $0.88(0.73-1.00)$ & Yes \\
\hline Nguyen (6) & 2015 & CA125 & NR & 10 & REM & 0.79 & $0.58(0.33-0.82)$ & $0.93(0.78-1.00)$ & Yes \\
\hline Liang (10) & 2008 & CA125 & $512 / 801$ & 10 & REM & 0.88 & $0.48(0.44-0.53)$ & $0.85(0.83-0.88)$ & Yes \\
\hline Nguyen (6) & 2015 & CA15-3 & NR & 11 & REM & 0.78 & $0.51(0.40-0.62)$ & $0.98(0.96-1.00)$ & Yes \\
\hline Wu (11) & 2015 & CA15-3 & $1,492 / 1,414$ & 21 & REM & 0.84 & $0.58(0.56-0.61)$ & $0.91(0.90-0.93)$ & Yes \\
\hline Liang (10) & 2008 & CA19-9 & $598 / 488$ & 7 & REM & 0.78 & $0.25(0.21-0.28)$ & $0.96(0.94-0.98)$ & Yes \\
\hline Nguyen (6) & 2015 & CYFRA 21-1 & NR & 16 & REM & 0.84 & $0.63(0.48-0.77)$ & $0.93(0.86-1.00)$ & Yes \\
\hline Liang (10) & 2008 & CYFRA 21-1 & $1,152 / 1,122$ & 18 & REM & 0.83 & $0.55(0.52-0.58)$ & $0.91(0.90-0.93)$ & Yes \\
\hline Gu (8) & 2007 & CYFRA 21-1 & $890 / 730$ & 12 & DLM & 0.82 & $0.47(0.44-0.51)$ & $0.92(0.90-0.94)$ & NR \\
\hline Yang (12) & 2017 & $\mathrm{CEA}+\mathrm{CA} 125$ & $107 / 167$ & 3 & REM & 0.86 & $0.65(0.56-0.74)$ & $0.98(0.94-0.99)$ & No \\
\hline Yang (12) & 2017 & $\mathrm{CEA}+\mathrm{CA} 153$ & $272 / 443$ & 4 & REM & 0.88 & $0.64(0.58-0.70)$ & $0.98(0.97-0.99)$ & No \\
\hline Yang (12) & 2017 & CEA+ CA19-9 & $264 / 355$ & 4 & REM & 0.96 & $0.58(0.51-0.64)$ & $0.98(0.96-0.99)$ & No \\
\hline Yang (12) & 2017 & CEA+CYFRA 21-1 & $325 / 415$ & 7 & REM & 0.95 & $0.82(0.77-0.86)$ & $0.92(0.89-0.95)$ & No \\
\hline
\end{tabular}

CEA, carcinoembryonic antigen; NSE, neuron-specific enolase; CA125, carbohydrate antigens 125; CA19-9, carbohydrate antigen 19-9; CA15-3, carbohydrate antigen; CYFRA 21-1, fragment of cytokeratin 19 (CYFRA 21-1); MPE, malignant pleural effusion; BPE, benign pleural effusion; N, number of included studies; NR, not reported; AUC, area under summary receiver operating characteristic curve; DLM, DerSimonian Laird method; REM, random-effects model; BVM, bivariate model; PB, publication bias.

has the inherent risk of operation-related complications (3). According to this evidence, development of non-invasive and objective diagnostic tools for MPE seems a promising perspective.

The measurement of some PE biomarkers has been proposed as a valuable approach for diagnosing MPE. Compared with PE cytology and pleural biopsy, diagnostic testing is mini-invasive, provides more objective results and is relatively less expensive (4). Furthermore, reliable and non-invasive testing allows early specialist referral, minimizes diagnostic delays, and optimizes clinical trials access (5).

To date, a number of biomarkers have been tested, and their diagnostic accuracy for MPE has been evaluated in a growing number of studies. Here, we provide a narrative review based on current scientific literature, aimed to summarize the current evidence of using pleural diagnostic biomarkers in MPE diagnostics. We present the following article in accordance with the Narrative Review reporting checklist (available at http://dx.doi.org/10.21037/tlcr-20-1111).

\section{Conventional cancer biomarkers}

To date, numerous studies have investigated the diagnostic accuracy of conventional cancer biomarkers for MPE, including carcinoembryonic antigen (CEA) (6-8), neuronspecific enolase (NSE) $(6,9)$, carbohydrate antigens 125 (CA125) (6,10), carbohydrate antigen 19-9 (CA19-9) $(6,10)$, carbohydrate antigen $15-3$ (CA15-3) $(6,10,11)$ and a fragment of cytokeratin 19 (CYFRA 21-1) $(6,8,10)$. Table 1 
summarizes the evidence from meta-analyses investigating the diagnostic accuracy of these cancer biomarkers. Generally, the overall sensitivity of traditional cancer biomarkers is unsatisfactory (around 0.50), while their specificities seem globally higher $(>0.90)$. Notably, nearly all the meta-analyses highlighted the presence of publication bias, thus indicating that the diagnostic accuracy of these conventional cancer biomarkers may be overestimated. A meta-analysis performed by Yang et al. in 2017 investigated the diagnostic accuracy for MPE of combined tumor markers (12) and reported that the combinations of positive pleural CEA + CA 19-9 and CEA + CA 15-3 had extremely high specificities for MPE (i.e., 0.99), but the sensitivity was low (around 0.65).

In another meta-analysis (13), the diagnostic accuracy of CEA, CA19-9, and CYFRA 21-1 for lung adenocarcinomaassociated MPE has been investigated. Among these tumor markers, CEA displayed the highest area under the curve (AUC; 0.93 ), and its sensitivity and specificity were 0.75 and 0.96, respectively. CA19-9 had 0.58 sensitivity, and 0.84 specificity, whilst CYFRA 21-1 exhibited 0.70 sensitivity and 0.88 specificity, respectively. These results indicate that the diagnostic accuracy of CEA, CA19-9, and CYFRA 21-1 for lung adenocarcinoma-associated MPE shall be considered overall modest.

Along with conventional cancer biomarkers earlier mentioned, some others have been investigated. Human epididymis protein 4 (HE4) has been for long considered a specific ovarian cancer biomarker, though a recent metaanalysis emphasized its moderate diagnostic accuracy for lung cancer (14). To date, two studies have investigated the diagnostic accuracy of HE4 for MPE $(15,16)$. In the first, the authors prospectively enrolled 32 patients with MPE and 54 patients with benign pleural effusion (BPE). They found that the AUC of pleural HE4 was 0.89 , with 0.85 sensitivity and 0.91 specificity at a diagnostic threshold of $1,675 \mathrm{pmol} / \mathrm{L}$ (16). In the second study, the authors investigated the diagnostic accuracy of HE4 for lung cancer-associated MPE (15) and found that pleural HE4 had an AUC of 0.83 for diagnosing MPE. At the $652.2 \mathrm{pmol} / \mathrm{L}$ threshold, the sensitivity and specificity of HE4 were 0.78 and 0.75 , respectively.

Four studies investigated the diagnostic accuracy of squamous cell carcinoma antigen (SCC) for diagnosing MPE (17-20). Two of these concluded that SCC concentration was not significantly increased in $\mathrm{MPE}$ $(17,20)$, whilst in two other studies, the diagnostic accuracy was found to be poor $(18,19)$. Notably, two of these studies also found that patients with squamous-cell lung cancer had considerably increased SCC values $(19,20)$. Taken together, these results suggest that the diagnostic accuracy of SCC is low for MPE, whilst this biomarker may have high diagnostic accuracy for squamous-cell lung cancerassociated MPE.

Progastrin-Releasing peptide (ProGRP) was proven as a useful diagnostic biomarker for small cell lung cancer (SCLC), with 0.64 sensitivity and 0.94 specificity, respectively (21). Only one study has analyzed the potential diagnostic utility of proGRP for MPE (22). In this study, the proGRP level in PE was found to be significantly higher in patients with SCLC, but its diagnostic accuracy was not assessed by statistical methods. In addition, no correlation was found between proGRP and NSE values, thus indicating that the combination of these two biomarkers may improve the overall diagnostic accuracy.

The ratio between $\mathrm{PE}$ and serum of cancer biomarkers can also be used for diagnosing MPE. In a prospective study including 98 patients with MPEs and 103 with BPEs, the authors investigated the diagnostic accuracy of pleural effusion CEA (pCEA) to serum CEA (sCEA) ratio (23). Surprisingly, it was found that the ratio displayed an AUC as high as 0.90 , with 0.85 sensitivity and 0.92 specificity at a threshold of 1.0, thus indicating that the $\mathrm{pCEA} / \mathrm{sCEA}$ ratio may represent a promising diagnostic tool.

\section{Vascular endothelial growth factor (VEGF) and its soluble receptor}

VEGF is a glycoprotein that acts as a promoter of angiogenesis, and is also critically involved in MPE formation. The first study investigating the diagnostic accuracy of VEGF for MPE has been published in 1993 (24). The authors found that the diagnostic sensitivity and specificity of VEGF in PE were 0.72 and 0.74 , respectively (24). Several other studies have then addressed the diagnostic role of PE VEGF in MPE, providing contradictory results $(25,26)$. In 2012 , a metaanalysis pooled the results of ten available studies, concluding that the diagnostic sensitivity and specificity of VEGF were 0.75 and 0.72 , respectively (27). Some other studies on this topic have since been published (28-31), so that an updated meta-analysis may be needed to re-assess the diagnostic accuracy of VEGF in MPE.

VEGF has three specific tyrosine kinase receptors, called VEGF receptor-1 (VEGFR1), VEGF receptor-2 (VEGFR2), and VEGF receptor-3 (VEGFR3) (32). VEGFR1, also known as Flt-1, is a negative angiogenesis regulator (32). The soluble form of VEGFR-1 (sVEGFR-1) 
has been identified in PE (33), with exudate displaying significantly higher sVEGFR-1values than transudate (33). To date, two studies have investigated the diagnostic accuracy of sVEGFR-1 in MPE. In the earlier study, including 40 patients with MPEs and 15 with BPEs, higher sVEGFR-1 value has been found in MPE. The AUC of sVEGFR-1 was as high as 0.93 (34), whilst sensitivity and specificity were 0.92 and 0.93 , respectively, using a $852 \mathrm{pg} / \mathrm{mL}$ diagnostic threshold (34). Nevertheless, the high diagnostic accuracy of sVEGFR-1 was not confirmed in a subsequent study (35), including 44 patients with MPEs and 36 with BPEs, whereby the AUC was found to be 0.79 , with 0.88 sensitivity and 0.58 specificity at a threshold of $3.95 \mathrm{ng} / \mathrm{mL}$ (35).

\section{Endostatin}

Unlike VEGF, which promotes angiogenesis, endostatin is an endogenous angiogenesis inhibitor, whose concentration has been associated with enhanced risk of developing various malignant diseases (36). The first report on the diagnostic accuracy of endostatin for diagnosing MPE has been published in 2003. In this study cohort, encompassing 38 patients with MPE and 29 with BPE, the sensitivity and specificity of this biomarker were 0.68 and 0.55 , respectively (37). In 2013, a meta-analysis was performed (38), concluding that the pooled sensitivity and specificity of endostatin for diagnosing MPE were 0.69 and 0.78 , respectively, thus underscoring that the overall diagnostic accuracy of this biomarker seems rather poor.

\section{Calprotectin}

In 2010, Rodríguez-Piñeiro et al. carried out a proteomic analysis for verifying the significance of some proteins for diagnosing MPE (39). Interestingly, S100-A8 and S100-A9, which form the non-covalent heterodimer, named calprotectin, were found to be decreased in MPE (39). An ensuing clinical trial was planned for evaluating the diagnostic accuracy of calprotectin in MPE, including 67 patients with MPE and 89 with BPE (40). It was finally found that calprotectin showed a considerably high diagnostic accuracy for MPE (AUC, 0.96). However, these results were not confirmed by three subsequent studies (41-43), which showed that the diagnostic accuracy (i.e., the AUC) of calprotectin in MPE was 0.68 (41), <0.50 (42), and 0.85 (43), respectively.
This results inconsistency may be attributable to the wide disease spectrum in the different study cohorts, as well as to the use of different calprotectin assays. Interestingly, in a recently published work, the authors found that calprotectin in transudate (defined by Light's criteria) was significantly lower than that in MPE (43), thus indicating that the proportion of transudate may have an impact on the diagnostic accuracy of this biomarker. In summary, more evidence would be needed to ascertain the diagnostic utility of calprotectin in PE.

\section{Cancer ratio and cancer ratio plus}

Cancer ratio, which is defined as serum lactate dehydrogenase (LDH) to $\mathrm{PE}$ adenosine deaminase ratio (ADA) ratio, has been firstly proposed by Verma et al., as a potential diagnostic parameter in MPE (44). The authors studied 100 patients with MPEs, 40 with TPE and 14 with PPE, reporting that the cancer ratio was increased in MPE, displaying an AUC of 0.81 for diagnosing MPE (44). This initial finding has then been validated in subsequent studies (45-47). A meta-analysis, published in 2019, concluded that sensitivity and specificity of cancer ratio were 0.97 and 0.89 , respectively (48). Nonetheless, all of the available studies were only based on PPE and/or TPE as control, whilst transudate was not included in the studied cohorts (48). Therefore, further studies using transudate would be necessary for better evaluating the diagnostic value of cancer ratio in MPE. Although HF due to PE is usually categorized into transudate by Light's criteria, misclassification as exudate could occur due to diuretic therapy (49), whilst nearly $5 \%$ of MPE can be misclassified into transudate (4). It is hence essential to include HF into the study cohort when investigating the diagnostic accuracy of MPE biomarkers.

Cancer ratio plus, which has also been proposed by Verma et al., is another diagnostic MPE parameter (45), defined as the ratio between cancer ratio and pleural lymphocyte count. To present, only one study has evaluated the diagnostic accuracy of cancer ratio plus in MPE. In this investigation, including 87 patients with MPEs and 34 with TPEs, the authors found an AUC of 0.86 for cancer ratio plus, which was higher than that of cancer ratio (AUC, 0.81). Using a cut-off of 50 , the sensitivity and specificity of cancer ratio plus were 0.89 and 0.94 , respectively, thus indicating that cancer ratio plus may also be seen as a useful diagnostic parameter in MPE. However, these results would also need to be validated in further studies, with larger sample sizes 
and more representative patients' cohorts.

\section{Homocysteine}

Homocysteine is an amino acid that is currently considered a risk factor for the development of cardiovascular and Alzheimer's diseases (50). In 2015, Santotoribio et al. firstly reported that homocysteine was increased in PE of patients with MPE (51). In a study cohort including PPE, MPE, TPE, and transudate, the AUC of homocysteine for diagnosing MPE was 0.83 , with 1.00 sensitivity and 0.57 specificity at $9.4 \mu \mathrm{mol} / \mathrm{L}$ threshold. These findings were replicated and validated in a subsequent study (52).

\section{Apolipoprotein E (Apo-E)}

Apo-E is a plasma lipoprotein that is usually considered a risk factor for cardiovascular disease, and some of its genetic polymorphisms have also been associated with increased risks of developing Alzheimer's disease (53). In a proteomic study performed in 2005, Apo-E was found to be higher in lung adenocarcinoma-induced MPE (54), indicating that this protein may retain a potential diagnostic value for MPE. In 2013, a prospective study investigated the diagnostic accuracy of Apo-E in MPE (55). In this study, including 160 patients with MPE and 40 with BPE, the AUC of Apo-E was 0.75 , whilst sensitivity and specificity were 0.88 and 0.86 , respectively, at $105 \mathrm{ng} / \mathrm{ml}$ threshold (55). Notably, since all MPE patients in this study were non-small-cell lung cancer (NSCLC), whether Apo-E may have similar diagnostic accuracy for MPE in patients with other etiology remains to be elucidated. Recently, Xue et al. enrolled a more representative patent cohort for investigating the diagnostic accuracy of Apo-E in MPE (56), obtaining a lower sensitivity (i.e., 0.79) and specificity (i.e., 0.74).

\section{Chitinase-like proteins (CLPs)}

CLPs play critical roles in various pulmonary and cardiovascular diseases such as asthma, chronic obstructive pulmonary disease (COPD) and HF $(57,58)$. Citinase-3-like protein 1 (YKL-40) and Citinase-3-like protein 2 (YKL-39) are the most investigated CLPs. Some studies explored the diagnostic accuracy of YKL-40 and YKL-39 for MPE, but the results are almost contradictory. One study found that the AUC of YKL-40 for MPE was 0.90 (59), though such a good diagnostic accuracy could not be replicated in other studies. For example, the AUC of YKL-40 for MPE in the study performed by Kayhan et al. was only 0.77 (60). In other two studies, YKL-40 was not even significantly increased in MPE $(61,62)$. The sources of heterogeneity among available studies were largely unknown. Taken together, these results would suggest that CLPs, including YKL-39 and YKL-40, may not be really useful diagnostic biomarkers in MPE, and this may be due to the fact that increased CLPs can be observed in many other diseases.

\section{B7 family}

The B7 family, which comprises a number of regulators of T-cell inhibition (63), has eleven members: B7-1 (CD80), B7-2 (CD86), B7-H1 (PD-L1), B7-DC (PD-L2), B7-H2 (ICOSL), B7-H3 (CD276), B7-H4 (VTCN1), B7-H5, BTNL2, B7-H6, and B7-H7. By binding to its ligand, the proteins of the $\mathrm{B} 7$ family regulate $\mathrm{T}$-cell proliferation and cytokine release. Some members are present in two forms, soluble and membrane-bound. The former was proven to be a diagnostic marker in various types of cancer. Some studies also investigated the diagnostic accuracy of soluble B7 family members for MPE.

Chen (64) et al. explored the diagnostic accuracy of soluble B7-H3 (sB7-H3) for MPE in 52 patients with NSCLC-derived MPE and 47 with BPE (28 exudates and 19 transudates), reporting an AUC of 0.85 for sB7-H3. The values of $\mathrm{sB} 7-\mathrm{H} 3$ in serum and $\mathrm{PE}$ were found to be positively correlated ( $\mathrm{r}=-0.784$ ), thus suggesting that $\mathrm{PE} \mathrm{sB} 7-\mathrm{H} 3$ may be passively diffused from blood to pleural cavity.

The diagnostic accuracy of soluble $\mathrm{B} 7-\mathrm{H} 4$ (sB7-H4) has also been investigated in two studies. In the first of these, encompassing 90 lung cancer patients with MPE and 58 with BPE (48 TPE and $10 \mathrm{PPE}$ ), the AUC of sB7-H4 was 0.862 (65). In the other study, Jing (66) enrolled a more representative cohort, consisting of 55 patients with MPEs due to various types of cancer and 42 with BPEs (25 TPEs, 7 PPEs, and $10 \mathrm{HFs}$ ), finally calculating an AUC of 0.884 for sB7-H4. A positive correlation was noted between the pleural values of $\mathrm{sB} 7-\mathrm{H} 4$ and $\mathrm{CEA}$, thus arguing that $\mathrm{sB} 7-\mathrm{H} 4$ may provide incremental diagnostic value beyond CEA.

\section{Matrix metalloproteinase (MMPs) and tissue- specific inhibitors of metalloproteinases (TIMPs)}

The MMPs are a family of extracellular matrix (ECM) degradation enzymes, consisting of 23 members, whose activity is counterbalanced by the TIMPs (67). MMPs and TIMPs are involved in various physiological and 
pathological processes, including the development of PE.

Among all the various members of the MMPs family, MMP-9 is the most studied. Notably, MMP-9 values were found to be increased in MPE in some studies (34,68-72), whilst no variation was found in another (73). Some studies also concluded that MMP-9 be increased in TPE (74-77) or PPE (78), but not in MPE. The possible sources of heterogeneity among these investigations may be attributable to the assay used for measuring MMP-9, to different disease spectrum of the study cohort, as well as to heterogeneous study design.

Two studies investigated the diagnostic accuracy of MMP-3 in MPE $(68,79)$. In one study, including 52 patients with malignant pleural mesothelioma (MPM), 33 with BPE, and 34 with lung cancer-induced MPE, MMP-3 was found to be increased in MPM, rather than in lung cancer-induced MPE (79). In another study, including 19 patients with lung cancer-induced MPE and 22 with BPE, MMP-3 was found to be increased in those with MPE (68). MMP-1 has also been studied in two studies, with conflicting results $(73,80)$. One study found that MMP-1 was higher in TPE than in transudate and MPE (80), while the other did not observe significant differences (73).

In addition to MMP-9, MMP-1, and MMP-3, the diagnostic accuracy of MMP-2 (73,74,78), MMP-7 (71), MMP-8 (73), TIMP-1 $(69,77)$, and TIMP-2 (77) has also been studied, and available results indicate that their diagnostic accuracy seems unsatisfactory, thus confirming that the diagnostic role of MMPs and TIMPs is probably very limited in MPE.

\section{Tumor-associated macrophages (TAMs) and monocytes}

There are many types of immune cells in the MPE environment, including lymphocytes, monocytes, and macrophages (81). Macrophages can be categorized into M1 and M2 types. M1 is a classically activated phenotype, and can produce pro-inflammatory and immunostimulatory cytokines that promote the clearance of tumor cell (81). M2 is instead an alternatively activated phenotype, which produces enzymes and cytokines suppressing the immune response against the tumor, thus potentially promoting cancer development (81). Two studies have investigated the diagnostic value of monocytes and macrophages in MPE.

In 2015, Wang et al. reported that the frequency of $\mathrm{CD} 14^{+} \mathrm{D} 163^{+}$macrophages in PE of MPE is approximately 10 -fold higher than that in BPE (82). The AUC of
$\mathrm{CD} 14^{+} \mathrm{D} 163^{+}$macrophages was considerably high (i.e., 0.941 ). At a threshold of $3.65 \%$, the diagnostic sensitivity and specificity of $\mathrm{CD} 14^{+} \mathrm{D} 163^{+}$macrophages were 0.86 and 1.00 , respectively (82). In another study, the authors investigated the diagnostic accuracy of CD206 ${ }^{+} \mathrm{CD} 14^{+}$ macrophages for lung cancer-induced MPE (83), reporting an AUC as high as 0.98 , with 0.88 sensitivity and 1.00 specificity at a $39.8 \%$ threshold.

Despite the results of these two studies are promising, their sample size was relatively modest (i.e., 60 and 100), whilst the diagnostic technique (i.e., flow cytometry) needs special training, is expensive, time-consuming, and poorly standardized.

\section{Reactive oxygen species modulator 1 (Romo1)}

Romo1 is a mitochondrial membrane protein involved in the production of intracellular reactive oxygen species (ROS) (84). It actively interplays with the development of various cancers, and increased serum Romol has been observed in lung cancer patients (85). Three studies have investigated the diagnostic accuracy of PE Romo1 for NSCLCassociated MPE so far (86-88), yielding AUCs between 0.71 and 0.84 , and thus underpinning a modest diagnostic accuracy. Notably, the MPE group in these studies only included NSCLC patients, so that future investigations with various types of MPE would be needed to evaluate the diagnostic accuracy of this putative biomarker.

\section{Epigenetic markers}

\section{$m R N A$}

In addition to protein and cellular markers, epigenetic biomarkers such as cell-free nucleic acid have been evaluated in MPE. To date, many studies have investigated the diagnostic accuracy of cell-free mRNA for MPE, and the results are summarized in Table 2. The diagnostic accuracy of available cell-free mRNAs is variegated. Overall, it seems that LUNX mRNA (89), MN/CA9 mRNA (90), MUC1 mRNA (91), and EpCAM mRNA (91) are more promising because, since their AUCs were $>0.90$, and their sensitivity and specificity were also $>0.90$.

\section{DNA methylation}

Some studies investigated the diagnostic accuracy of DNA methylation in MPE. In a study performed by Katayama et al. (100) using methylation-specific polymerase chain 
Table 2 Summary of studies investigating the diagnostic accuracy of cell-free mRNA for MPE

\begin{tabular}{|c|c|c|c|c|c|c|c|c|c|}
\hline Author & Year & Country & Markers & Design & MPE/non-MPE & Non-MPE & AUC & Sensitivity & Specificity \\
\hline \multirow[t]{2}{*}{ Sun (90) } & 2014 & China & MUC1 mRNA & NR & $58 / 40$ & PPE, TPE, HF & 0.92 & 0.67 & 0.95 \\
\hline & & & EpCAM mRNA & NR & $58 / 40$ & PPE, TPE, HF & 0.92 & 0.71 & 0.95 \\
\hline Li (91) & 2007 & France & MN/CA9 mRNA & Prospective & $59 / 12$ & NR & NR & 0.90 & 0.92 \\
\hline Tang (93) & 2015 & China & Foxm mRNA & NR & $23 / 15$ & PPE, TPE, HF & 0.88 & 0.83 & 0.87 \\
\hline Li (94) & 2013 & China & p16 mRNA loss & Prospective & $58 / 46$ & PPE, TPE, HF, O & NR & 0.48 & 0.90 \\
\hline \multirow[t]{2}{*}{ Bao (95) } & 2014 & China & LUNX mRNA & Prospective & $76 / 60$ & PPE, TPE, HF, LC, O & 0.78 & 0.52 & 0.95 \\
\hline & & & VEGF mRNA & Prospective & $76 / 60$ & PPE, TPE, HF, LC, O & 0.80 & 0.68 & 0.95 \\
\hline Yu (97) & & & MUC5AC mRNA & Prospective & $54 / 35$ & PPE, TPE & NR & 0.72 & 0.96 \\
\hline Jeon (98) & 2012 & Korea & MAGE mRNA & Prospective & $44 / 23$ & PPE, TPE, HF, O & NR & 0.61 & 0.96 \\
\hline Li (99) & 2012 & China & XAIP mRNA & NR & $56 / 42$ & PPE, TPE, HF, LC, O & 0.76 & 0.66 & 0.86 \\
\hline
\end{tabular}

NR, not reported; MPE, malignant pleural effusion; PPE, parapneumonic effusion; TPE, tuberculous pleural effusion; HF, heart failure; LC, liver cirrhosis; O, others; AUC, area under curve.

reaction, the authors assessed the aberrant hypermethylation of DNA repair gene ras association domain family $1 \mathrm{~A}$ (RASSF1A), O6-methylguanine-DNA methyltransferase (MGMT), p16INK4a, apoptosis-related genes, retinoic acid receptor $\mathrm{b}(\mathrm{RARb})$, death-associated protein kinase (DAPK). Among these five epigenetic biomarkers, DAPK was unrelated to MPE. The incorporation of the remaining four biomarkers into a logistic regression model yielded sensitivity and specificity of 0.60 and 0.79 , respectively. Benlloch et al. (101) studied the diagnostic accuracy of these four epigenetic markers with a parallel diagnostic approach (at least one gene methylated in pleural fluid) and obtained 1.00 specificity, counterbalanced by 0.59 sensitivity. Some studies have also investigated the role of methylation of SHOX2 (102), SEPT9 (102), and WIF-1 promoter region (103) in MPE diagnostics. Although their specificity was as high as 1.00 , sensitivities were almost unsatisfactory, thus raising doubts as to whether DNA methylation may be a useful diagnostic tool in MPE.

\section{Cell-free microRNA}

MicroRNAs are short non-coding RNAs that regulate gene expression at the post-transcriptional level (104).
microRNAs are sufficiently stable in the circulation and have been suggested as potential diagnostic markers for various diseases, including cancer (105) and tissue injury $(106,107)$. The first report concerning the diagnostic accuracy of pleural microRNA in MPE diagnostics has been published in 2010 (108). The authors measured the level of 22 cell-free miRNAs, which were found to be dysregulated in lung cancer patients' serum. miR-24, miR-26a, and miR$30 \mathrm{~d}$ were increased in MPE and ascites. The diagnostic accuracy of miR-24 and miR-30d in MPE was also evaluated (109), with AUCs of 0.71 and 0.75, respectively.

In 2013, a study exploring a miRNA array revealed that miR-98 was a possible diagnostic biomarker for lung adenocarcinoma-associated malignant pleural effusion (LA-MPE) (110). Unlike traditional cancer biomarkers, which are increased in PE, miR-98 was decreased in LAMPE. The AUC of miR-98 for LA-MPE was 0.887 , which is comparable to the diagnostic performance of CEA and CYFRA 21-1. A logistic regression model, incorporating CEA, CYFRA 21-1, and miR-98, displayed a considerably high AUC (i.e., 0.926), thus indicating that miR-98 can improve the diagnostic accuracy of CEA and CYFRA 21-1. In a subsequent study, PE miR-134, miR-185, and miR-22 were found to be decreased in LA-MPE patients (111). The 
AUCs of these three microRNAs were between 0.72 and 0.83 , and their combination with CEA was found to improve the diagnostic accuracy of LA-MPE (AUC, 0.942). In 2018, a study based on 20 MPEs and 20 BPEs analyzed the diagnostic value of miR-21 and miR-24 in MPE. The AUCs of these two microRNAs were approximately 0.87 (112).

\section{Exosomal microRNA}

The source of cell-free microRNA in PE remains largely unknown. For circulating microRNA, three hypotheses have been proposed to explain their origin (113), including passive released by injured cells, active release in the form of a complex with proteins such as high-density lipoproteins (HDL), and release in extracellular vesicles (EVs) such as exosomes and microvesicles. It hence seems that these three hypotheses can also be used to explain the source of cellfree microRNA in PE. The cell-free microRNAs can be directly released into the pleural cavity by tumor cells or passively diffuse from blood to the pleural cavity.

Exosomes are EVs secreted by all types of cells, with a diameter between 30 and $100 \mathrm{~nm}$ (114). Because miRNA signatures in circulating exosomes are very similar to those originating from tumor cells (115), they appear to be more specific cancer biomarkers. Moreover, exosomal microRNAs seem more stable than the other two forms of cell-free microRNA, because their encapsulation within the exosome prevents their possible degeneration (116).

Two studies with microRNA array have addressed the differentially expressed exosomal microRNAs in MPE and BPE $(117,118)$. Some microRNAs could be identified, such as miR-375 and miR-200. The diagnostic accuracy of exosomal microRNAs was assessed with receiver operating characteristics (ROC) curve analysis in the former investigation (118), and the AUCs of miR-375, miR-200b, miR-200c, and miR-141 were found to be al $>0.90$. The second study explored the diagnostic accuracy of exosomal miR-21, miR-31, miR-182, and miR210 for MPE (119). Among these, the diagnostic accuracy of miR-182 (AUC, 0.87) and miR-210 (AUC, 0.81) was the most promising. Unfortunately, the MPE group in these three studies only included lung adenocarcinoma and the sample size was small, so that the diagnostic accuracy of exosomal microRNAs in MPE would need to be evaluated in future clinical investigations.

\section{Tumor markers for MPM}

$\mathrm{PE}$ is relatively common in MPM patients. It is reported that nearly $3 \%$ of undiagnosed $\mathrm{PE}$ are MPM, and PE can be observed in about half of MPM patients $(120,121)$. Therefore, PE biomarkers represent a promising diagnostic tool for MPM. Soluble mesothelin-related peptides (SMRP), osteopontin, and fibulin-3 are the most widely studied biomarkers for MPM (122), and the diagnostic role of their serum or plasma concentrations have been explored in several meta-analyses (123-125). However, the diagnostic value of their PE concentrations remains largely unknown. Only two studies have investigated the possible diagnostic role of PE SMRP for MPM $(126,127)$, providing contradictory findings. The sensitivity, specificity and AUC in one study were 0.79 (95\% CI: $0.75-0.83$ ), 0.85 (95\% CI: $0.83-0.87$ ) and 0.89 (95\% CI: 0.85-0.93), respectively. In the second study, sensitivity specificity and AUC were $0.69(95 \%$ CI: $0.64-0.72), 0.90$ (95\% CI: $0.85-0.94)$ and 0.76 (95\% CI: $0.72-0.80)$, respectively. The proteomic technology also shows high diagnostic accuracy for MPM (128), with one study finding an AUC as high as 0.99. However, additional evidence would be needed to support these preliminary data.

Recent studies have also highlighted that cell-free microRNA in PE may be seen as a novel diagnostic marker for MPM. However, according to a recently published literature review (129), the diagnostic accuracy of PE microRNA is limited, and more studies will be needed to validate.

\section{Conclusions}

In this review, we have summarized several cancer biomarkers, whose assessment may be useful in MPE diagnosis. Some of these, such as cytotoxic T lymphocyteassociated antigen-4 (CTLA-4) (130), Her 2 (131), total sialic acid (TSA) (132), carbohydrate antigen 549 (CA 549) (133), tissue polypeptide-specific (TPS) (134), midkine (46), syndecan-1 and osteopontin (135), endothelial-cell-specific molecule-1 (ESM-1) (136) and heat shock protein $90 \beta$ (HSP90 $\beta$ ) (137) have been investigated in a limited number of studies, which also failed to provide encouraging results. TAMs seem to have the highest diagnostic accuracy, but their assessment is expensive, time-consuming, requires skilled personnel and test results are poorly standardized. Cancer ratio represents another promising parameter in MPE (48), but future studies would be needed to validate its diagnostic accuracy.

Notably, some of the above-mentioned studies may be plagued by a patients' selection bias. For example, only lung 
cancer patients were enrolled in the MPE group, and thus the disease spectrum may not be representative enough of the common malignancies causing $\mathrm{PE}$ in clinical practice. Moreover, subjects' enrollment in some studies was not consecutive, and this may have led to a selection bias (138).

We can hence conclude that the diagnostic accuracy of each single cancer biomarker is relatively modest so that it seems reasonable to suggest that a multi-marker strategy may be a much better approach in MPE diagnostics. Although the development of such algorithms is indeed challenging, artificial intelligence approaches could be an option $(139,140)$. Further studies, such as SIMPLE (141) and DIAPHRAGM (5), are attempting to use this approach for improving the diagnostic accuracy of cancer biomarkers in MPE. In the promising era of targeted cancer treatment, therapeutic strategies are determined by therapy-related genetic alterations, such as EGFR mutation. Unfortunately, the current evidence does not support PE soluble markers for predicting the genetic alteration. Under such a circumstance, invasive biopsy using an appropriate specimen is unavoidable. Therefore, exploring soluble biomarkers that can predict the therapy-related genetic alteration is an attractive object for future studies.

\section{Acknowledgments}

Funding: This work is supported by the National Natural Science Foundation of China (81860501); and the Natural and Science Foundation of Inner Mongolia Autonomous Region for Distinguished Young Scholars (2020JQ07).

\section{Footnote}

Reporting Checklist: The authors have completed the Narrative Review reporting checklist. Available at http:// dx.doi.org/10.21037/tlcr-20-1111

Conflicts of Interest: All authors have completed the ICMJE uniform disclosure form (available at http://dx.doi. org/10.21037/tlcr-20-1111). The authors have no conflicts of interest to declare.

Ethical Statement: The authors are accountable for all aspects of the work in ensuring that questions related to the accuracy or integrity of any part of the work are appropriately investigated and resolved.

Open Access Statement: This is an Open Access article distributed in accordance with the Creative Commons Attribution-NonCommercial-NoDerivs 4.0 International License (CC BY-NC-ND 4.0), which permits the noncommercial replication and distribution of the article with the strict proviso that no changes or edits are made and the original work is properly cited (including links to both the formal publication through the relevant DOI and the license). See: https://creativecommons.org/licenses/by-nc-nd/4.0/.

\section{References}

1. Porcel JM, Azzopardi M, Koegelenberg CF, et al. The diagnosis of pleural effusions. Expert Rev Respir Med 2015;9:801-15.

2. Loveland P, Christie M, Hammerschlag G, et al. Diagnostic yield of pleural fluid cytology in malignant effusions: an Australian tertiary centre experience. Intern Med J 2018;48:1318-24.

3. Wang XJ, Yang Y, Wang Z, et al. Efficacy and safety of diagnostic thoracoscopy in undiagnosed pleural effusions. Respiration 2015;90:251-5.

4. Thomas JM, Musani AI. Malignant pleural effusions: a review. Clin Chest Med 2013;34:459-71.

5. Tsim S, Kelly C, Alexander L, et al. Diagnostic and Prognostic Biomarkers in the Rational Assessment of Mesothelioma (DIAPHRAGM) study: protocol of a prospective, multicentre, observational study. BMJ Open 2016;6:e013324.

6. Nguyen AH, Miller EJ, Wichman CS, et al. Diagnostic value of tumor antigens in malignant pleural effusion: a meta-analysis. Transl Res 2015;166:432-9.

7. Shi HZ, Liang QL, Jiang J, et al. Diagnostic value of carcinoembryonic antigen in malignant pleural effusion: a meta-analysis. Respirology 2008;13:518-27.

8. Gu P, Huang G, Chen Y, et al. Diagnostic utility of pleural fluid carcinoembryonic antigen and CYFRA 21-1 in patients with pleural effusion: a systematic review and meta-analysis. J Clin Lab Anal 2007;21:398-405.

9. Zhu J, Feng M, Liang L, et al. Is neuron-specific enolase useful for diagnosing malignant pleural effusions? evidence from a validation study and meta-analysis. BMC Cancer 2017;17:590.

10. Liang QL, Shi HZ, Qin XJ, et al. Diagnostic accuracy of tumour markers for malignant pleural effusion: a metaanalysis. Thorax 2008;63:35-41.

11. Wu Q, Li M, Zhang S, et al. Clinical diagnostic utility of CA 15-3 for the diagnosis of malignant pleural effusion: A meta-analysis. Exp Ther Med 2015;9:232-8. 
12. Yang Y, Liu YL, Shi HZ. Diagnostic Accuracy of Combinations of Tumor Markers for Malignant Pleural Effusion: An Updated Meta-Analysis. Respiration 2017;94:62-9.

13. Feng $M, Z$ hu J, Liang $L$, et al. Diagnostic value of tumor markers for lung adenocarcinoma-associated malignant pleural effusion: a validation study and meta-analysis. Int J Clin Oncol 2017;22:283-90.

14. Yan L, Hu ZD. Diagnostic accuracy of human epididymis secretory protein 4 for lung cancer: a systematic review and meta-analysis. J Thorac Dis 2019;11:2737-44.

15. Lv M, Wang F, Wang X, et al. Diagnostic value of human epididymis protein 4 in malignant pleural effusion in lung cancer. Cancer Biomark 2019;26:523-8.

16. Elsammak MY, Attia A, Hassan HA, et al. Evaluation of pleural fluid human epididymis 4 (HE4) as a marker of malignant pleural effusion. Tumour Biol 2012;33:1701-7.

17. Ferrer J, Villarino MA, Encabo G, et al. Diagnostic utility of CYFRA 21-1, carcinoembryonic antigen, CA 125, neuron specific enolase, and squamous cell antigen level determinations in the serum and pleural fluid of patients with pleural effusions. Cancer 1999;86:1488-95.

18. San Jose ME, Alvarez D, Valdes L, et al. Utility of tumour markers in the diagnosis of neoplastic pleural effusion. Clin Chim Acta 1997;265:193-205.

19. Liu L, Liu B, Zhu LL, et al. Clinical significance of CYFRA21-1, Scc-Ag and telomerase activity in serum and pleural effusion of patients with squamous-cell lung cancer. Bioanalysis 2012;4:2367-74.

20. Miédougé M, Rouzaud P, Salama G, et al. Evaluation of seven tumour markers in pleural fluid for the diagnosis of malignant effusions. Br J Cancer 1999;81:1059-65.

21. Wang H, Qian J. Serum pro-gastrin-releasing peptide in diagnosis of small cell lung cancer: A meta-analysis. J Cancer Res Ther 2016;12:C260-3.

22. Shijubo N, Hirasawa M, Sasaki H, et al. Elevated progastrin-releasing peptide(31-98) concentrations in pleural effusions due to small-cell lung carcinoma. Respiration 1996;63:106-10.

23. Hackner K, Errhalt P, Handzhiev S. Ratio of carcinoembryonic antigen in pleural fluid and serum for the diagnosis of malignant pleural effusion. Ther Adv Med Oncol 2019;11:1758835919850341.

24. Yeo KT, Wang HH, Nagy JA, et al. Vascular permeability factor (vascular endothelial growth factor) in guinea pig and human tumor and inflammatory effusions. Cancer Res 1993;53:2912-8.

25. Zhou WB, Bai M, Jin Y. Diagnostic value of vascular endothelial growth factor and endostatin in malignant pleural effusions. Int J Tuberc Lung Dis 2009;13:381-6.

26. Shu J, Sun G, Liu H, et al. Clinical utility of vascular endothelial growth factor in diagnosing malignant pleural effusions. Acta Oncol 2007;46:1004-11.

27. Shen YC, Liu MQ, Wan C, et al. Diagnostic accuracy of vascular endothelial growth factor for malignant pleural effusion: A meta-analysis. Exp Ther Med 2012;3:1072-6.

28. Psatha A, Makris D, Kerenidi T, et al. A potential role for VEGF in the diagnostic approach of pleural effusions. J Thorac Dis 2016;8:1681-7.

29. Wu DW, Chang WA, Liu KT, et al. Vascular endothelial growth factor and protein level in pleural effusion for differentiating malignant from benign pleural effusion. Oncol Lett 2017;14:3657-62.

30. Kim HR, Kim BR, Park RK, et al. Diagnostic Significance of Measuring Vascular Endothelial Growth Factor for the Differentiation between Malignant and Tuberculous Pleural Effusion. Tohoku J Exp Med 2017;242:137-42.

31. Gu Y, Zhang M, Li GH, et al. Diagnostic values of vascular endothelial growth factor and epidermal growth factor receptor for benign and malignant hydrothorax. Chin Med J (Engl) 2015;128:305-9.

32. Shibuya $M$. Vascular endothelial growth factor receptor-1 (VEGFR-1/Flt-1): a dual regulator for angiogenesis. Angiogenesis 2006;9:225-30; discussion 31.

33. Tomimoto H, Yano S, Muguruma H, et al. Levels of soluble vascular endothelial growth factor receptor 1 are elevated in the exudative pleural effusions. J Med Invest 2007;54:146-53.

34. Fiorelli A, Morgillo F, Fasano M, et al. The value of matrix metalloproteinase-9 and vascular endothelial growth factor receptor 1 pathway in diagnosing indeterminate pleural effusion. Interact Cardiovasc Thorac Surg 2013;16:263-9.

35. Zhu YY, Wu HM, Liu RY. Diagnostic Values of sVEGFR-1 and Endostatin in Malignant Pleural Effusions in Patients with Lung Cancer. Clin Lab 2017;63:1371-8.

36. Marneros AG, Olsen BR. Physiological role of collagen XVIII and endostatin. FASEB J 2005;19:716-28.

37. Sumi M, Kagohashi K, Satoh H, et al. Endostatin levels in exudative pleural effusions. Lung 2003;181:329-34.

38. Tian P, Shen $Y$, Feng $M$, et al. Diagnostic accuracy of endostatin for malignant pleural effusion: A clinical study and meta-analysis. Postgrad Med 2015;127:529-34.

39. Rodríguez-Piñeiro AM, Blanco-Prieto S, Sánchez-Otero $\mathrm{N}$, et al. On the identification of biomarkers for nonsmall cell lung cancer in serum and pleural effusion. J Proteomics 2010;73:1511-22. 
40. Sanchez-Otero N, Blanco-Prieto S, Paez de la Cadena M, et al. Calprotectin: a novel biomarker for the diagnosis of pleural effusion. Br J Cancer 2012;107:1876-82.

41. Luo J, Wang M, Li C, et al. A Novel Combination of Calprotectin and CXCL12 for Predicting Malignancy in Patients with Exudative Pleural Effusion. Medicine (Baltimore) 2015;94:e2105.

42. Faria DK, Faria CS, Doi D, et al. Hybrid panel of biomarkers can be useful in the diagnosis of pleural and peritoneal effusions. Clin Chim Acta 2019;497:48-53.

43. Botana-Rial M, Vazquez-Iglesias L, Casado-Rey P, et al. Validation of Calprotectin As a Novel Biomarker For The Diagnosis of Pleural Effusion: a Multicentre Trial. Sci Rep 2020;10:5679.

44. Verma A, Abisheganaden J, Light RW. Identifying Malignant Pleural Effusion by A Cancer Ratio (Serum LDH: Pleural Fluid ADA Ratio). Lung 2016;194:147-53.

45. Verma A, Dagaonkar RS, Marshall D, et al. Differentiating Malignant from Tubercular Pleural Effusion by Cancer Ratio Plus (Cancer Ratio: Pleural Lymphocyte Count). Can Respir J 2016;2016:7348239.

46. Zhang F, Wang J, Zheng X, et al. Clinical value of jointly detection pleural fluid Midkine, pleural fluid adenosine deaminase, and pleural fluid carbohydrate antigen 125 in the identification of nonsmall cell lung cancerassociated malignant pleural effusion. J Clin Lab Anal 2018;32:e22576.

47. Korczynski P, Mierzejewski M, Krenke R, et al. Cancer ratio and other new parameters for differentiation between malignant and nonmalignant pleural effusions. Pol Arch Intern Med 2018;128:354-61.

48. Han YQ, Zhang L, Yan L, et al. Diagnostic accuracy of cancer ratio for malignant pleural effusion: a systematic review and meta-analysis. Ann Transl Med 2019;7:554.

49. Bielsa S, Porcel JM, Castellote J, et al. Solving the Light's criteria misclassification rate of cardiac and hepatic transudates. Respirology 2012;17:721-6.

50. Cacciapuoti F. Lowering homocysteine levels with folic acid and B-vitamins do not reduce early atherosclerosis, but could interfere with cognitive decline and Alzheimer's disease. J Thromb Thrombolysis 2013;36:258-62.

51. Santotoribio JD, Canavate-Solano C, Garcia-de la Torre A, et al. Homocysteine: new tumor marker in pleural fluid. Tumour Biol 2015;36:7941-5.

52. Santotoribio JD, Del Valle-Vazquez L, Garcia-de la Torre A, et al. The diagnostic value of pleural fluid homocysteine in malignant pleural effusion. PLoS One 2019;14:e0222616.
53. Mahley RW. Apolipoprotein E: from cardiovascular disease to neurodegenerative disorders. J Mol Med (Berl) 2016;94:739-46.

54. Tyan YC, Wu HY, Lai WW, et al. Proteomic profiling of human pleural effusion using two-dimensional nano liquid chromatography tandem mass spectrometry. J Proteome Res 2005;4:1274-86.

55. Wang Y, Chen Z, Chen J, et al. The diagnostic value of apolipoprotein $\mathrm{E}$ in malignant pleural effusion associated with non-small cell lung cancer. Clin Chim Acta 2013;421:230-5.

56. Xue Q, Fang G, Deng X, et al. The value of apolipoprotein $\mathrm{E}$ in distinguishing benign and malignant unilateral pleural effusions. Medicine (Baltimore) 2020;99:e19320.

57. Tong X, Wang D, Liu S, et al. The YKL-40 protein is a potential biomarker for COPD: a meta-analysis and systematic review. Int J Chron Obstruct Pulmon Dis 2018;13:409-18.

58. Kastrup J. Can YKL-40 be a new inflammatory biomarker in cardiovascular disease? Immunobiology 2012;217:483-91.

59. Javath Hussain S, Selvaraj J, Mohanty Mohapatra M, et al. Clinical utility of pleural fluid YKL-40 as a marker of malignant pleural effusion. Curr Probl Cancer 2019;43:354-62.

60. Kayhan S, Gumus A, Cinarka H, et al. The clinical utility of pleural YKL-40 levels in diagnosing pleural effusions. J Thorac Dis 2013;5:634-40.

61. Adamidi T, Soulitzis N, Neofytou E, et al. Expression of YKL-40 and MIP-1a proteins in exudates and transudates: biomarkers for differential diagnosis of pleural effusions? A pilot study. BMC Pulm Med 2015;15:150.

62. Kim HR, Jun CD, Lee KS, et al. Levels of YKL-40 in pleural effusions and blood from patients with pulmonary or pleural disease. Cytokine 2012;58:336-43.

63. Bour-Jordan H, Esensten JH, Martinez-Llordella M, et al. Intrinsic and extrinsic control of peripheral T-cell tolerance by costimulatory molecules of the CD28/ B7 family. Immunol Rev 2011;241:180-205.

64. Chen L, Zhang G, Sheng S, et al. Upregulation of soluble B7-H3 in NSCLC-derived malignant pleural effusion: A potential diagnostic biomarker correlated with NSCLC staging. Clin Chim Acta 2016;457:81-5.

65. Xu C, Qian L, Yu L, et al. Evaluation of serum and pleural levels of soluble B7-H4 in lung cancer patients with pleural effusion. Biomarkers 2015;20:271-4.

66. Jing X, Wei F, Li J, et al. Diagnostic value of soluble B7-H4 and carcinoembryonic antigen in distinguishing 
malignant from benign pleural effusion. Clin Respir J 2018;12:986-90.

67. Cui N, Hu M, Khalil RA. Biochemical and Biological Attributes of Matrix Metalloproteinases. Prog Mol Biol Transl Sci 2017;147:1-73.

68. Kremer R, Best LA, Savulescu D, et al. Pleural fluid analysis of lung cancer vs benign inflammatory disease patients. Br J Cancer 2010;102:1180-4.

69. Fiorelli A, Ricci S, Feola A, et al. Matrix metalloproteinase- 9 and tissue inhibitor of metalloproteinase-1 in diagnosis of pleural effusion of malignant origin. Interact Cardiovasc Thorac Surg 2016;22:411-8.

70. Jin HY, Lee KS, Jin SM, et al. Vascular endothelial growth factor correlates with matrix metalloproteinase-9 in the pleural effusion. Respir Med 2004;98:115-22.

71. Cheng D, Liang B, Li YH. Application of MMP-7 and MMP-10 in assisting the diagnosis of malignant pleural effusion. Asian Pac J Cancer Prev 2012;13:505-9.

72. Carpagnano GE, Palladino GP, Martinelli D, et al. Exhaled matrix metalloproteinase-9 in lung cancer. Rejuvenation Res 2012;15:359-65.

73. Teixeira LR, Dias MB, Sales RK, et al. Profile of Metalloproteinases and Their Association with Inflammatory Markers in Pleural Effusions. Lung 2016;194:1021-7.

74. Hsieh WY, Kuan TC, Cheng KS, et al. ACE/ACE2 ratio and MMP-9 activity as potential biomarkers in tuberculous pleural effusions. Int J Biol Sci 2012;8:1197-205.

75. Park KJ, Hwang SC, Sheen SS, et al. Expression of matrix metalloproteinase-9 in pleural effusions of tuberculosis and lung cancer. Respiration 2005;72:166-75.

76. Sheen P, O'Kane CM, Chaudhary K, et al. High MMP-9 activity characterises pleural tuberculosis correlating with granuloma formation. Eur Respir J 2009;33:134-41.

77. Vatansever S, Gelisgen R, Uzun H, et al. Potential role of matrix metalloproteinase-2,-9 and tissue inhibitors of metalloproteinase-1,-2 in exudative pleural effusions. Clin Invest Med 2009;32:E293-300.

78. Oikonomidi S, Kostikas K, Kalomenidis I, et al. Matrix metalloproteinase levels in the differentiation of parapneumonic pleural effusions. Respiration 2010;80:285-91.

79. Murakami A, Tabata C, Tabata R, et al. Clinical role of pleural effusion MMP-3 levels in malignant pleural mesothelioma. Oncol Lett 2012;3:581-5.

80. Hong JY, Park SY, Kim Y, et al. Calpain and spectrin breakdown products as potential biomarkers in tuberculous pleural effusion. J Thorac Dis 2018;10:2558-66.

81. Pathria P, Louis TL, Varner JA. Targeting TumorAssociated Macrophages in Cancer. Trends Immunol 2019;40:310-27.

82. Wang F, Yang L, Gao Q, et al. CD163+CD14+ macrophages, a potential immune biomarker for malignant pleural effusion. Cancer Immunol Immunother 2015;64:965-76.

83. Pei XB, Wu XZ, Yi FS, et al. Diagnostic value of CD206(+)CD14(+) macrophages in diagnosis of lung cancer originated malignant pleural effusion. J Thorac Dis 2019;11:2730-6.

84. Chung YM, Kim JS, Yoo YD. A novel protein, Romo1, induces ROS production in the mitochondria. Biochem Biophys Res Commun 2006;347:649-55.

85. Lee SH, Lee JS, Lee EJ, et al. Serum reactive oxygen species modulator 1 (Romo1) as a potential diagnostic biomarker for non-small cell lung cancer. Lung Cancer 2014;85:175-81.

86. Lee SH, Park MJ, Choi SI, et al. Reactive oxygen species modulator 1 (Romo1) as a novel diagnostic marker for lung cancer-related malignant effusion. Medicine (Baltimore) 2017;96:e5975.

87. Chen X, Zhang N, Dong J, et al. Reactive oxygen species modulator 1 , a novel protein, combined with carcinoembryonic antigen in differentiating malignant from benign pleural effusion. Tumour Biol 2017;39:1010428317698378.

88. Zhang F, Wang J, Fu J, et al. Clinical value of combined detection of reactive oxygen species modulator 1 and adenosine deaminase in pleural effusion in the identification of NSCLC associated malignant pleural effusion. J Clin Lab Anal 2020;34:e23091.

89. Tang Y, Xu L. Superiority and clinical significance of Lunx mRNA in the diagnosis of malignant pleural effusion caused by pulmonary carcinoma. J Exp Clin Cancer Res 2013;32:37.

90. Sun W, Li J, Jiang HG, et al. Diagnostic value of MUC1 and EpCAM mRNA as tumor markers in differentiating benign from malignant pleural effusion. QJM 2014;107:1001-7.

91. Li G, Passebosc-Faure K, Feng G, et al. MN/CA9: a potential gene marker for detection of malignant cells in effusions. Biomarkers 2007;12:214-20.

92. Shu J, Sun GY, Liu AP, et al. Diagnostic accuracy of human telomerase reverse transcriptase mRNA in malignant pleural effusions: A preliminary report for in situ hybridization detection. Clin Chim Acta 2007;381:131-5. 
93. Tang Z, Li H, Zhu H, et al. Different expression of FoxM1 in human benign and malignant pleural effusion. Med Oncol 2015;32:312.

94. Li J, Bao QL, Wang Y, et al. Diagnostic value of the FHIT and p16 mRNA loss and the K-ras gene mutation in pleural fluids for malignant pleural effusion. Cancer Biomark 2013;13:49-58.

95. Bao QL, Li J, Sun W, et al. Diagnostic utility of LUNX mRNA and VEGF mRNA in pleural fluid for differentiating benign from malignant origin. Jpn J Clin Oncol 2014;44:1198-205.

96. Jiang B, Wu GP, Zhao YJ, et al. Transcription expression and clinical significance of TTF-1 mRNA in pleural effusion of patients with lung cancer. Diagn Cytopathol 2008;36:849-54.

97. Yu CJ, Shew JY, Liaw YS, et al. Application of mucin quantitative competitive reverse transcription polymerase chain reaction in assisting the diagnosis of malignant pleural effusion. Am J Respir Crit Care Med 2001;164:1312-8.

98. Jeon EJ, Park HK, Jeon K, et al. The role of pleural fluid MAGE RT-nested PCR in the diagnosis of malignant pleural effusion. Thorac Cancer 2012;3:320-5.

99. Li J, Li ZN, Bao QL, et al. Evaluation of pleural fluid survivin and XIAP for the diagnosis of malignant pleural effusion. Tumour Biol 2012;33:1803-10.

100. Katayama H, Hiraki A, Aoe K, et al. Aberrant promoter methylation in pleural fluid DNA for diagnosis of malignant pleural effusion. Int J Cancer 2007;120:2191-5.

101.Benlloch S, Galbis-Caravajal JM, Martin C, et al. Potential diagnostic value of methylation profile in pleural fluid and serum from cancer patients with pleural effusion. Cancer 2006;107:1859-65.

102.Dietrich D, Jung M, Puetzer S, et al. Diagnostic and prognostic value of SHOX2 and SEPT9 DNA methylation and cytology in benign, paramalignant and malignant pleural effusions. PLoS One 2013;8:e84225.

103. Yang TM, Leu SW, Li JM, et al. WIF-1 promoter region hypermethylation as an adjuvant diagnostic marker for non-small cell lung cancer-related malignant pleural effusions. J Cancer Res Clin Oncol 2009;135:919-24.

104. Bartel DP. MicroRNAs: target recognition and regulatory functions. Cell 2009;136:215-33.

105. Bartels CL, Tsongalis GJ. MicroRNAs: novel biomarkers for human cancer. Clin Chem 2009;55:623-31.

106.Ji X, Takahashi R, Hiura Y, et al. Plasma miR-208 as a biomarker of myocardial injury. Clin Chem 2009;5 5:1944-9.
107.Laterza OF, Lim L, Garrett-Engele PW, et al. Plasma MicroRNAs as sensitive and specific biomarkers of tissue injury. Clin Chem 2009;55:1977-83.

108.Xie L, Chen X, Wang L, et al. Cell-free miRNAs may indicate diagnosis and docetaxel sensitivity of tumor cells in malignant effusions. BMC Cancer 2010;10:591.

109.Xie L, Wang T, Yu S, et al. Cell-free miR-24 and miR-30d, potential diagnostic biomarkers in malignant effusions. Clin Biochem 2011;44:216-20.

110.Han HS, Yun J, Lim SN, et al. Downregulation of cell-free miR-198 as a diagnostic biomarker for lung adenocarcinoma-associated malignant pleural effusion. Int J Cancer 2013;133:645-52.

111. Shin YM, Yun J, Lee OJ, et al. Diagnostic Value of Circulating Extracellular miR-134, miR-185, and miR22 Levels in Lung Adenocarcinoma-Associated Malignant Pleural Effusion. Cancer Res Treat 2014;46:178-85.

112.Liu C, Huang L, Zhang X, et al. Combination of DNA ploidy analysis and miR-21 or miR-24 in screening malignant pleural effusion. Interact Cardiovasc Thorac Surg 2018;26:376-81.

113. Wojczakowski W, Kobylarek D, Lindner J, et al. MicroRNAs - novel biomarkers for malignant pleural effusions. Contemp Oncol (Pozn) 2019;23:133-40.

114.Zhou L, Lv T, Zhang Q, et al. The biology, function and clinical implications of exosomes in lung cancer. Cancer Lett 2017;407:84-92.

115. Rabinowits G, Gercel-Taylor C, Day JM, et al. Exosomal microRNA: a diagnostic marker for lung cancer. Clin Lung Cancer 2009;10:42-6.

116. Cortez MA, Bueso-Ramos C, Ferdin J, et al. MicroRNAs in body fluids--the mix of hormones and biomarkers. Nat Rev Clin Oncol 2011;8:467-77.

117. Wang Y, Xu YM, Zou YQ, et al. Identification of differential expressed $\mathrm{PE}$ exosomal miRNA in lung adenocarcinoma, tuberculosis, and other benign lesions. Medicine (Baltimore) 2017;96:e8361.

118.Hydbring P, De Petris L, Zhang Y, et al. Exosomal RNAprofiling of pleural effusions identifies adenocarcinoma patients through elevated miR-200 and LCN2 expression. Lung cancer (Amsterdam, Netherlands) 2018;124:45-52.

119. Tamiya H, Mitani A, Saito A, et al. Exosomal MicroRNA Expression Profiling in Patients with Lung Adenocarcinoma-associated Malignant Pleural Effusion. Anticancer Res 2018;38:6707-14.

120.Porcel JM, Esquerda A, Vives M, et al. Etiology of pleural effusions: analysis of more than 3,000 consecutive thoracenteses. Arch Bronconeumol 2014;50:161-5. 
121. Woolhouse I, Bishop L, Darlison L, et al. British Thoracic Society Guideline for the investigation and management of malignant pleural mesothelioma. Thorax 2018;73:i1-i30.

122. Chen Z, Gaudino G, Pass HI, et al. Diagnostic and prognostic biomarkers for malignant mesothelioma: an update. Transl Lung Cancer Res 2017;6:259-69.

123.Hu ZD, Liu XF, Liu XC, et al. Diagnostic accuracy of osteopontin for malignant pleural mesothelioma: a systematic review and meta-analysis. Clin Chim Acta 2014;433:44-8.

124.Luo L, Shi HZ, Liang QL, et al. Diagnostic value of soluble mesothelin-related peptides for malignant mesothelioma: a meta-analysis. Respir Med 2010;104:149-56.

125. Ren R, Yin P, Zhang Y, et al. Diagnostic value of fibulin-3 for malignant pleural mesothelioma: A systematic review and meta-analysis. Oncotarget 2016;7:84851-9.

126. Cui A, Jin XG, Zhai K, et al. Diagnostic values of soluble mesothelin-related peptides for malignant pleural mesothelioma: updated meta-analysis. BMJ Open 2014;4:e004145.

127. Gao R, Wang F, Wang Z, et al. Diagnostic value of soluble mesothelin-related peptides in pleural effusion for malignant pleural mesothelioma: An updated metaanalysis. Medicine (Baltimore) 2019;98:e14979.

128. Ostroff RM, Mehan MR, Stewart A, et al. Early detection of malignant pleural mesothelioma in asbestosexposed individuals with a noninvasive proteomics-based surveillance tool. PLoS One 2012;7:e46091.

129. Han YQ, Xu SC, Zheng WQ, et al. Diagnostic value of microRNAs for malignant pleural mesothelioma: A minireview. Thorac Cancer 2021;12:8-12.

130. Chen M, Xie S, Wan C, et al. Diagnostic performance of CTLA-4, carcinoembryonic antigen and CYFRA 21-1 for malignant pleural effusion. Postgrad Med 2017;129:644-8.

131. Hung TL, Chen FF, Liu JM, et al. Clinical evaluation of HER-2/neu protein in malignant pleural effusion-associated lung adenocarcinoma and as a tumor marker in pleural effusion diagnosis. Clin Cancer Res 2003;9:2605-12.

132. Alatas F, Alatas O, Metintas M, et al. Diagnostic value of CEA, CA 15-3, CA 19-9, CYFRA 21-1, NSE and TSA assay in pleural effusions. Lung Cancer 2001;31:9-16.

133.Hernandez L, Espasa A, Fernandez C, et al. CEA and CA 549 in serum and pleural fluid of patients with pleural effusion. Lung Cancer 2002;36:83-9.

134. Villena V, Echave-Sustaeta J, Lopez-Encuentra A, et al. Determination of tissue polypeptide-specific antigen in pleural fluid and serum from patients with pleural effusion. Int J Biol Markers 1995;10:161-5.

135.Mundt F, Heidari-Hamedani G, Nilsonne G, et al. Diagnostic and prognostic value of soluble syndecan- 1 in pleural malignancies. Biomed Res Int 2014;2014:419853.

136.Lu GJ, Shao CJ, Zhang Y, et al. Diagnostic and prognostic values of endothelial-cell-specific molecule-1 with malignant pleural effusions in patients with non-small cell lung cancer. Oncotarget 2017;8:49217-23.

137. Biaoxue R, Min L, Tian F, et al. Elevated Hsp90-beta contributes to differential diagnosis of pleural effusion caused by lung cancer and correlates with malignant biological behavior of lung cancer. BMC Pulm Med 2018;18:188.

138.Zhang M, Hu ZD. Suggestions for designing studies investigating diagnostic accuracy of biomarkers. Ann Transl Med 2019;7:788.

139.Li C, Hou L, Sharma BY, et al. Developing a new intelligent system for the diagnosis of tuberculous pleural effusion. Comput Methods Programs Biomed 2018;153:211-25.

140.Ren Z, Hu Y, Xu L. Identifying tuberculous pleural effusion using artificial intelligence machine learning algorithms. Respir Res 2019;20:220.

141.Han YQ, Yan L, Li P, et al. A Study Investigating Markers in PLeural Effusion (SIMPLE): a prospective and doubleblind diagnostic study. BMJ Open 2019;9:e027287.
Cite this article as: Zhang M, Yan L, Lippi G, Hu ZD. Pleural biomarkers in diagnostics of malignant pleural effusion: a narrative review. Transl Lung Cancer Res 2021;10(3):15571570. doi: $10.21037 /$ tlcr-20-1111 